\section{MR Angiography of a 1-Year-Old Girl with Semilobar Holoprosencephaly}

Holoprosencephaly encompasses a variety of brain malformations and is thought to be a result of failure of cleavage of the prosencephalon into the diencephalon and telencephalon and/or stemming from a defect in the normal induction and patterning of the rostral neural tube, which normally occurs in the first 4 weeks after conception. ${ }^{1}$ We present a case of semilobar holoprosencephaly in a 1-year-old girl, with emphasis on the arterial vasculature demonstrated by MR angiographic (MRA) imaging.

A 1-year-old girl with a history of developmental delay presented with failure to thrive and decreased oral intake. Physical examination showed a microcephalic infant with right microphthalmos, bilateral colobomas, and poor muscle strength and tone. MRA of the head demonstrated paired internal carotid arteries, each of which bifurcated in a roughly symmetric manner. The bilateral bifurcating vessels supplied the frontoparietal cortices and probably represented analogues of the anterior and middle cerebral arteries. The segmental arteries coursed anteriorly and laterally but did not cross the midline; there was no anterior communicating artery. The posterior circulation was unremarkable, and a left posterior communicating artery was present (Figs 1 and 2).

Holoprosencephaly has classically been categorized as lobar, semilobar, or alobar, in order of increasing severity of manifestation. Although the anatomic features of holoprosencephaly have been well described, the accompanying anomalous cerebral vasculature has only been sporadically demonstrated in the literature via angiographic case reports ${ }^{2}$ and schematics from an autopsy study. ${ }^{3}$ van Overbeeke et $\mathrm{al}^{3}$ described 3 different vascular patterns in the anterior part of the circle of Willis in the autopsy study. The first pattern (type A) is essentially normal, with a closed anterior part of the circle of Willis. The second pattern (type B) describes roughly symmetric paired internal carotid arteries supplying the cortical surface, giving rise to smaller vessels, but failing to cross the midline; no anterior communicating artery is present. The third pattern (type C) describes

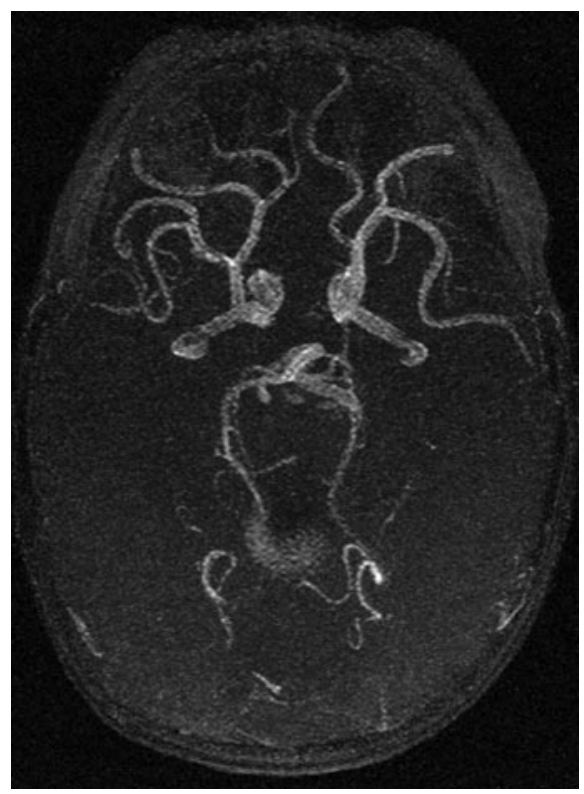

Fig 1. Axial view of maximum intensity projection from 3D time-of-flight MR angiogram of the head demonstrates paired internal carotid arteries bifurcating into segmental arteries, representing analogues of the anterior and middle cerebral arteries. No anterior communicating artery is present. The posterior circulation is unremarkable.

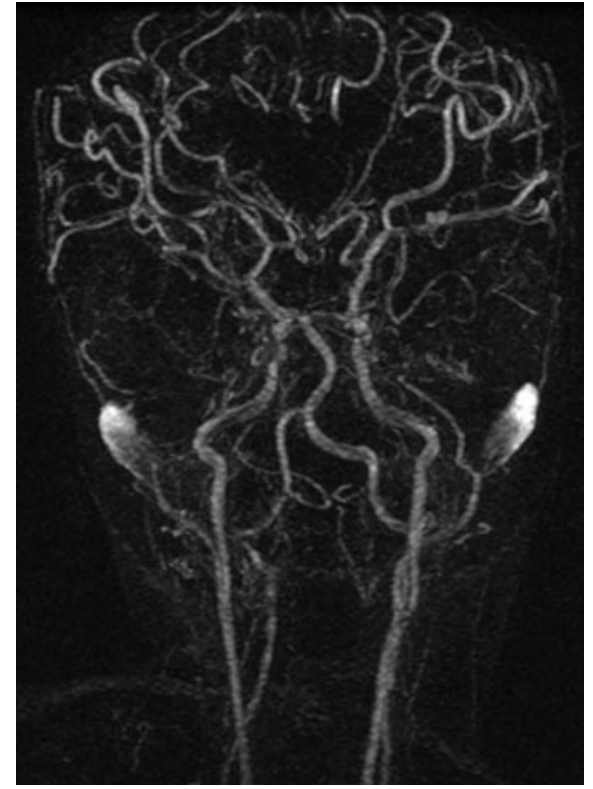

Fig 2. Coronal view of maximum intensity projection from gadolinium MR angiogram of the head and neck demonstrates paired internal carotid arteries bifurcating into segmental arteries, representing analogues of the anterior and middle cerebral arteries.

a dominant internal carotid artery and an azygous anterior cerebral artery, which supplies most of the cortical surface. The 3 patterns roughly correlate with the degree of holoprosencephalic severity, with type A occurring in arrhinencephalic brains and type $\mathrm{C}$ occurring in both semilobar and alobar holoprosencephalic brains. The vascular pattern shown in this case study corresponds to the type B vascular pattern.

The failure of prosencephalon cleavage leading to holoprosencephaly occurs at 3 to 4 weeks after conception, whereas the development of anterior and middle cerebral arteries occurs at approximately 4 to 7 weeks. ${ }^{4}$ Therefore, the anomalous vascular patterns found in holoprosencephalic brains likely reflect an adaptation of embryologic cerebrovascular growth to early anatomic malformations in the developing brain. Specifically, the anterior communicating artery forms at 7 weeks from plexiform anastomoses between the 2 anterior cerebral arteries. The failure of prosencephalon cleavage likely results in nonformation of the anterior communicating artery because of aberrant anatomy of the underlying brain parenchyma.

\section{References}

1. Golden JA. Towards a greater understanding of the pathogenesis of holoprosencephaly. Brain Develop 1999;21:513-21

2. Maki Y, Kumagai K. Angiographic features of alobar holoprosencephaly. Neuroradiology 1974;6:270-76

3. van Overbeeke JJ, Hillen M, Vermeij-Keers $\mathrm{CH}$. The arterial pattern at the base of arhinencephalic and holoprosencephalic brains. J Anat 1994; 185:51-63

4. Okahara M, Kiyosue $\mathrm{H}$, Mori $\mathrm{H}$, et al. Anatomic variations of the cerebral arteries and their embryology: a pictorial review. Eur Radiology 2002; $12: 2548-61$

C.K. Byun

K.H. Chao

M.S. Tenner

J.Y. Hu

G.K. Paek

New York Medical College

Department of Radiology

Valhalla, NY 